\title{
Spherical nanometer-sized diamond obtained from detonation
}

\author{
P.W. Chen ${ }^{\mathrm{a}, *}$, Y.S. Ding ${ }^{\mathrm{a}}$, Q. Chen ${ }^{\mathrm{b}}$, F.L. Huang ${ }^{\mathrm{c}}$, S.R. Yun ${ }^{\mathrm{c}}$ \\ ${ }^{a}$ Division 2, Institute of Mechanics, Chinese Academy of Sciences, Beijing 100080, PR China \\ ${ }^{\mathrm{b}}$ Institute of Applied Physics and Computational Mechanics, Beijing 100088, PR China \\ ${ }^{\mathrm{c}}$ Department of Safety Engineering, Beijing Institute of Technology, Beijing 100081, PR China
}

\begin{abstract}
Ultrafine diamond (UFD) was synthesized under high pressure and high temperatures generated by explosive detonation. The structure, composition, surface and thermal stability of UFD were studied by use of XRD, TEM, Raman Spectroscopy, FTIR, etc. The influences of the synthesis conditions and purification conditions on the properties of UFD were analyzed. The UFD had an average size of 4-6 nm, commonly exhibiting a spherical shape. The highest yield was of up to 10 mass $\%$ of the explosive. Attempts were made to use UFD as an additive to metal-diamond sintering and as crystallite seeds of CVD diamond films. The results show that UFD can decrease the coefficient of friction of the composite by $30 \%$, and raise the nucleation density in CVD diamond films by 2-3 times. (c) 2000 Elsevier Science S.A. All rights reserved.
\end{abstract}

Keywords: Ultrafine diamond; Detonation; Characterization; Application

\section{Introduction}

The synthesis of ultrafine diamond by the detonation of explosives with a negative oxygen balance appeared in the 1980s [1,2]. At present, the investigation of UFD under detonation conditions is being quickly developed in several scientific centers. The mechanism of the synthesis of UFD under detonation conditions remains controversial. Some new application fields distinguished from those of normal diamond are emerging. The present paper has presented generalized results of the properties of UFD prepared in our laboratory. Some preliminary results have also been presented for using UFD as an additive to metal-diamond sintering and as crystallite seeds of CVD diamond films.

The explosive used in the experiment was a mixture of TNT and RDX. The charges were detonated in a steel chamber with a volume of $1.6 \mathrm{~m}^{3}$. The mass of

\footnotetext{
* Corresponding author.

E-mail address: pwchen@imech.ac.cn (P.W. Chen).
}

charge was approximately $200 \mathrm{~g}$. In order to preserve the diamond phase and to reduce the presence of non-diamond phases, the chamber was filled with inert gases $\left(\mathrm{N}_{2}, \mathrm{CO}_{2}, \mathrm{Ar}\right.$, etc.), or else the charges were wrapped in water, ice or pyrolytic salts before detonation. After detonation, the UFD was separated from the black carbon soot containing diamond, graphite and amorphous carbon. Besides commonly used perchloric acid and a mixture of nitric acid and sulfuric acid, $\mathrm{KMnO}_{4}$ was used to purify UFD. The results show that $\mathrm{KMnO}_{4}$ is a more efficient, safer, cheaper and less polluting oxidizing agent. When purified by $\mathrm{KMnO}_{4}$, the cost of UFD can be noticeably reduced by $50 \%$, compared with that by perchloric acid [3].

\section{Characterization}

Synthesis conditions and purification conditions can influence the elemental composition of UFD. According to the elemental analysis, the UFD contained: carbon, 87-90\%; hydrogen, 0.5-1.0\%; nitrogen, 


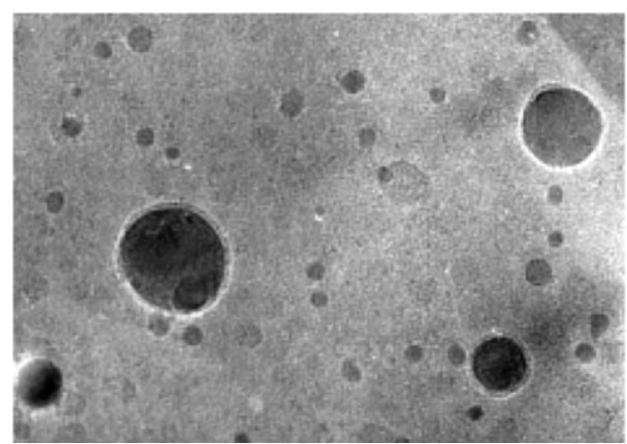

Fig. 1. High resolution TEM micrograph of UFD.

1.6-2.5\%; and oxygen, 6-10\%. XPS analysis showed that $83 \%$ of carbon atoms were in the form of $\mathrm{sp}^{3}, 5 \%$ in $\mathrm{sp}^{2}$, and $12 \%$ in $\mathrm{C}-\mathrm{H}, \mathrm{C}-\mathrm{O}$ and $\mathrm{C}-\mathrm{N}$, suggesting that some unbonded carbon atoms on the surface of UFD were saturated by N, H and $\mathrm{O}$ atoms. Small angle $\mathrm{X}$-ray scattering (SAXS) analysis showed that the grain size range was $1-50 \mathrm{~nm}$ and the average grain size was 4-6 nm. The specific area measured up to $420 \mathrm{~m}^{2} / \mathrm{g}$.

A high-resolution TEM (HTEM) examination (Fig. 1) showed that the UFD had a round shape and practically no sharp angles, which ensured that UFD was an ideal additive for antifriction and antiwear use. The larger particles were in a size range of $10-20 \mathrm{~nm}$, and the smaller ones 4-6 $\mathrm{nm}$. The round shape of UFD could be explained as the result of crystallization from liquid or liquid-like carbon during detonation. This special morphology was also observed by Greiner et al. [2] and Mal'kov et al. [4].

The X-ray diffraction analysis of UFD obtained under different conditions is shown in Fig. 2. Three peaks, corresponding to (111), (220), (311) diffraction of diamond, demonstrated that UFD had a cubic conformation. The three peaks had considerable width due to the small size effects of the crystals. A minor peak at approximately $2 \theta=26^{\circ}$ (see curve 2 ) and broad diffraction bands in the range of $2 \theta=20-30^{\circ}$ (see curves 1 and 2) showed the presence of graphite and amorphous carbon after purification. As indicated in Table 1, UFD was characterized by having considerable microstress and larger crystal lattice parameters than bulk diamond. Such a high level of lattice deformation could be explained either by the inhomogeneous deformation of UFD particles during the strongly non-equilibrium detonation process, or by the effect of other inserted atoms in the crystal lattice. The value of UFD microstress did not change noticeably when the UFD was heated up to $1000^{\circ} \mathrm{C}$ in an $\mathrm{Ar}$ atmosphere, which can be explained by the high potential barrier hindering the annealing of defects.

Raman spectra were measured at room temperature with the 514.5-nm line of an argon ion laser [5]. Fig. 3 shows the Raman spectrum of UFD obtained under the preservation of water. The Raman spectrum of bulk diamond is given for comparison. The Raman bands assigned to $\mathrm{sp}^{2}$ and $\mathrm{sp}^{3}$ carbon were observed at approximately 1600 and $1323 \mathrm{~cm}^{-1}$, respectively, suggesting that UFD contained a small amount of $\mathrm{sp}^{2}$ carbon. The broad Raman band at approximately $400-700 \mathrm{~cm}^{-1}$ was assigned to amorphous carbon. The Raman band assigned to $\mathrm{sp}^{3}$ carbon was asymmetric with a shift of $-8.9 \mathrm{~cm}^{-1}$ and a linewidth of $30.4 \mathrm{~cm}^{-1}$. These results were consistent with those of Yoshikawa [6].

The Fourier-IR spectrum (Fig. 4) shows that the UFD surface was covered by a large amount of carbonyl, carboxyl, methyl and nitryl groups. Due to this fact, it was possible to chemically modify the UFD surface. The synthesis and purification conditions could have affected the type and amount of groups on the

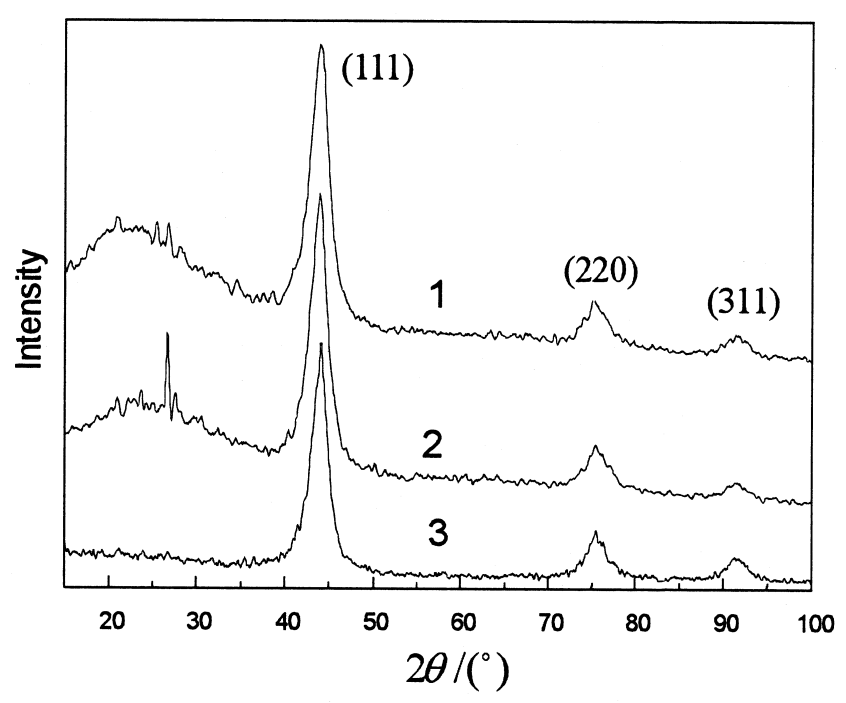

Fig. 2. X-Ray diffraction analysis of UFD obtained under preserving conditions and purified by $\mathrm{HClO}_{4}$ at $190^{\circ} \mathrm{C}$ for $4 \mathrm{~h}$. (1) $=\mathrm{N} 2$; (2) $=$ pyrolytic salt; (3) = water.

Table 1

Microstructure of UFD

\begin{tabular}{llll}
\hline Preserving conditions & $\begin{array}{l}\text { Average deviation } \\
\text { of cell parameters } \\
(\%)\end{array}$ & $\begin{array}{l}\text { Average grain size } \\
(\mathrm{nm}) \\
\text { (Wilson method) }\end{array}$ & $\begin{array}{l}\text { Microstress } \\
(\%)\end{array}$ \\
\hline $\mathrm{N}_{2}$ & +0.25 & 4.2 & 0.6381 \\
Pyrolytic salt & +0.09 & 3.7 & 0.410 \\
Water & +0.24 & 6.6 & 1.004 \\
\hline
\end{tabular}




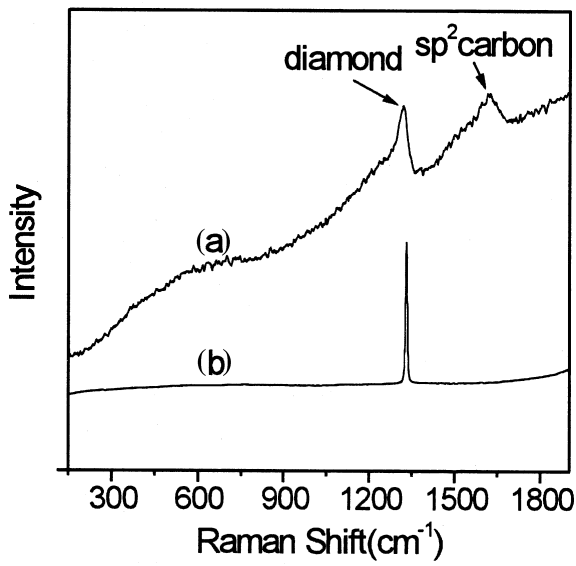

Fig. 3. Raman spectrum of UFD obtained under the preservation of water and purified by $\mathrm{HClO}_{4}$ at $190^{\circ} \mathrm{C}$ for $4 \mathrm{~h}$. (a) = UFD; (b) = bulk diamond.

surface of UFD. The presence of these functional groups could cause the deviation of the lattice parameter and the density of UFD from the corresponding values for normal diamond crystals.

Due to the active surface and strong adsorption activity, UFD particles were aggregated into clusters of dimensions from hundreds of nanometers to several micrometers. The clusters could exhibit round or polyhedral shapes. Highly dispersed and stabilized suspensions of UFD could be obtained through selecting the appropriate liquid media and dispersing agents.

Thermal analysis was carried out by TG and DTA. On heating in air at a rate of $15^{\circ} \mathrm{C} \mathrm{min}^{-1}$, UFD, obtained under the preservation of water and purified by $\mathrm{HClO}_{4}$, started oxidation at $528^{\circ} \mathrm{C}$, and its loss of weight at $625^{\circ} \mathrm{C}$ was $50 \%$. On heating in an Ar atmosphere at the same rate, the speed of oxidation was

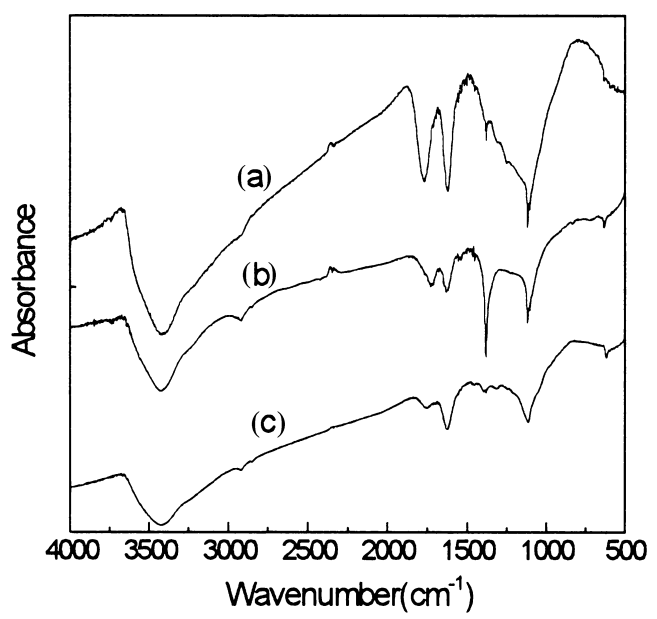

Fig. 4. FTIR spectra of UFD obtained from different synthesis and purification conditions: (a) under the preservation of $\mathrm{CO}_{2}$ and purified by $\mathrm{HClO}_{4}$; (b) under the preservation of water and purified by $\mathrm{HClO}_{4}$; and (c) under the preservation of water and purified by $\mathrm{KMnO}_{4}$.

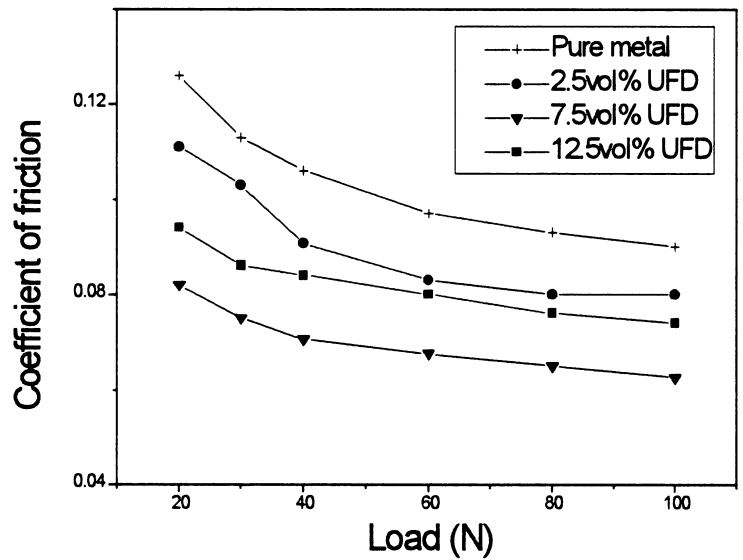

Fig. 5. Coefficients of friction as a function of the applied load of UFD-metal composite for different UFD content.

noticeably slower. The loss of weight was $7.55 \%$ at $706^{\circ} \mathrm{C}$ and $22.4 \%$ at $1000^{\circ} \mathrm{C}$. UFDs obtained under the preservation of ice, $\mathrm{N}_{2}$ and $\mathrm{CO}_{2}$ started their oxidation at 505,500 and $515^{\circ} \mathrm{C}$, respectively. Of all the preserving media that we used, water generated the best thermal stability. When the preservation conditions remained the same, the oxidization starting temperature of samples purified by $\mathrm{KMnO}_{4}$ was $8^{\circ} \mathrm{C}$ higher than that under $\mathrm{HClO}_{4}$. In reducing atmosphere $\mathrm{CO}$, the thermal stability of UFD could also be noticeably enhanced; its oxidation starting temperature was $200^{\circ} \mathrm{C}$ higher than in air.

\section{Application}

Due to its round shape, UFD could generate particularly favorable slip conditions in a friction couple. It has even been thought to satisfy the requirement for rolling friction through the rotation of UFD particles on a nanometer scale, although not on a molecular scale [7]. UFD, copper, zinc and tin were sintered to fabricate anti-friction composites through hot pressing sintering. The sintering temperature and pressure were $800^{\circ} \mathrm{C}$ and $18 \mathrm{MPa}$, respectively. Experiments were carried to evaluate the antifriction behavior of the composites. XRD analysis showed that the grain size and microstress of UFD remained almost unchanged during sintering. Fig. 5 shows the coefficients of friction of the composites as a function of the applied load. The results show that when the content of UFD was approximately $7.5 \mathrm{vol} \%$, the composite possessed the best antifriction behavior, with a decrease in the coefficient of friction of $30 \%$, compared with that of pure metal composite. Further study is underway to investigate the mechanism of antifriction and if there exists nano-ball bearing effects of UFD clusters, as reported by Ouyang et al. [7]. 


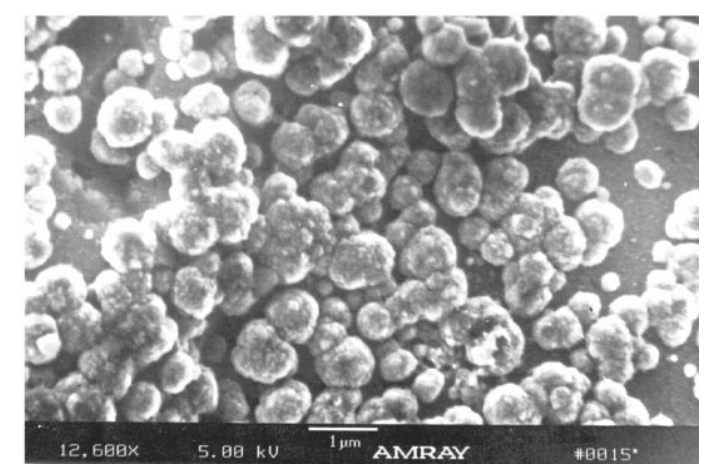

Fig. 6. SEM micrograph of the Mo substrate after $10 \mathrm{~min}$ deposition.

UFD could also be used as crystallite seeds to improve the nucleation density in CVD diamond film. These experiments were carried out by using a DC plasma jet apparatus [8]. Fig. 6 shows a SEM micrograph of a UFD-pasted Mo substrate after $10 \mathrm{~min}$ deposition. Fig. 7 shows a SEM micrograph of the CVD diamond film deposited on the UFD-pasted Mo after 2 $\mathrm{h}$ deposition. The results show that the nucleation density could be raised 2-3 times (up to $3.3-4 \times 10^{8}$ $\mathrm{cm}^{2}$ ) by pasting the substrate with UFD particles. The modality of crystallites on the film also showed differences to those of films obtained by the regular CVD method. Further investigations will be focussed on the chemical modification of the UFD surface and keeping its reactive activity.

Our research on the application of UFD also involved electrodepositing metal-UFD coating, abrasive polishing paste and explosion consolidation. The application of UFD could also be expanded to other fields. For example, It could be used as an effective antifric-

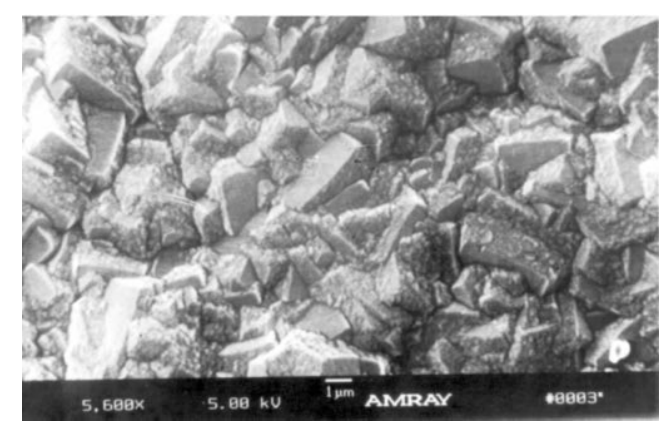

Fig. 7. SEM micrograph of the CVD diamond film deposited on UFD pasted substrate after $2 \mathrm{~h}$ deposition. tion and antiabrasive additive to mineral oil, and as a highly effective absorbent. To expand the fields of the application of UFD and to improve the quality of UFD products, special attention should be paid to the deaggragation of UFD clusters and to the physical and chemical modification of UFD.

\section{Conclusions}

Detonation-synthesized spherical UFD possesses a variety of properties which help to distinguish it from the known different types of synthetic diamond. Control of the synthesis conditions enables UFD to possess the prescribed physiochemical properties. The high yield (up to 10 mass $\%$ of the explosive) ensures a fairly low cost. Its nanometric particle size, unique spherical shape, stable physiochemical properties in neutral and reducing atmospheres, and low cost open a series of application fields.

\section{Acknowledgements}

The authors of this paper acknowledge financial support from the Chinese National Natural Science Foundation.

\section{References}

[1] A.M. Staver, N.V. Gubareva, A.I. Lyamkin et al., Fizika Goreniya I Vzryza 20 (5) (1984).

[2] N.R. Greiner, D.S. Philips, J.D. Johnson et al., Diamonds in detonation soot, Nature 333 (1988) 440-442.

[3] P.W. Chen, Ultrafine diamond obtained by detonation: theory and characterization. PhD Dissertation, Beijing Institute of Technology, (1999).

[4] I.Y. Mal'kov, L.I. Filatov, V.M. Titov et al., Formation of diamond from liquid carbon, Fizika Goreniya I Vzryza 29 (4) (1993) 131-134.

[5] P.W. Chen, S.R. Yun, F.L. Huang et al., Raman scattering of ultrafine diamond obtained from detonation, Chin. J. High Pressure Phys. 13 (1) (1999) 59-63.

[6] M. Yoshikawa, Y. Mori, H. Obata et al., Appl. Phys. Lett. 67 (5) (1995).

[7] Q. Ouyang, K. Okada, Appl. Surf. Sci. 78 (1994) 309-313.

[8] Q. Chen, Synthesizing ultrafine diamond: theory and application research. PhD Dissertation, Beijing Institute of Technology, (1998). 